\title{
SOME TRACE FORMULAS FOR ALMOST UNPERTURBED SCHRÖDINGER PAIRS OF OPERATORS
}

\author{
DAOXING XIA
}

(Communicated by Paul S. Muhly)

\begin{abstract}
Some trace formulas are given for an almost unperturbed Schrödinger pair of operators $\{U, V\}$ and a self-adjoint operator $V_{0}$, where $V_{0}$ satisfies the condition that $\left\{U, V_{0}\right\}$ is a Schrödinger pair of operators and $\left\{V, V_{0}\right\}$ is almost commuting.
\end{abstract}

\section{INTRODUCTION}

This paper is a continuation of previous works [5] and [6]. Let $\mathscr{H}$ be a Hilbert space and $\{U, V\}$ be a pair of self-adjoint operators on $\mathscr{H}$. This pair $\{U, V\}$ is said to be a Schrödinger pair of operators if

$$
e^{i s U} e^{i V t} e^{-i U s} e^{-i V t}=e^{i s t} I, \quad-\infty<s, t<+\infty .
$$

A condition equivalent to (1) is

$$
i\left[(U-\lambda I)^{-1},(V-\mu I)^{-1}\right]=(U-\lambda I)^{-1}(V-\mu I)^{-2}(U-\lambda I)^{-1}
$$

for some $\lambda, \mu \in \mathbb{C} \backslash \mathbb{R}$. A pair of self-adjoint operators is said to be an almost unperturbed Schrödinger pair of operators if there is a trace class operator $D$ such that

$$
i\left[(U-\lambda I)^{-1},(V-\mu I)^{-1}\right]=(U-\lambda I)^{-1}(V-\mu I)^{-1}(I+D)(V-\mu I)^{-1}(U-\lambda I)^{-1} \text {. }
$$

(cf. [5, p. 243] with $a=1$ ). For this pair $\{U, V\}$, a cyclic cocycle is given by

$$
\begin{aligned}
& \operatorname{tr}\left(\left[e^{i s_{1} U} e^{i t_{1} V}, e^{i s_{2} U} e^{i t_{2} V}\right]-e^{i\left(s_{1}+s_{2}\right) U} e^{i\left(t_{1}+t_{2}\right) V}\left(e^{-i s_{2} t_{1}}-e^{-i s_{1}, t_{2}}\right)\right) \\
& =\tau\left(s_{1}+s_{2}, t_{1}+t_{2}\right)\left(e^{-i s_{2} t_{1}}-e^{-i t_{1} s_{2}}\right),
\end{aligned}
$$

where $[\cdot, \cdot]$ is the commutator, and the function $\tau$ may be written

$$
\tau(s, t)=\operatorname{tr}\left(e^{i s U} \int_{0}^{t} e^{i \tau U} D e^{i(t-\tau) V} d \tau\right) / t .
$$

Received by the editors January 2, 1990 and, in revised form, March 19, 1990; presented to the G.P.O.T.S, Albuquerque, New Mexico, April 19, 1990.

1980 Mathematics Subject Classification (1985 Revision). Primary 47A35; Secondary 47G05.

Key words and phrases. Trace formula, cyclic cocycle, Schrödinger pair of operators, perturbation.

This work was supported in part by NSF grant DMS- 8901506 . 
Under certain conditions, the operator $V$ in the almost unperturbed Schrödinger operator $\{U, V\}$ is a perturbation of the operator $V_{0}$ in the Schrödinger pair $\left\{U, V_{0}\right\}$ (see [5, Theorem 2]). Therefore, in this paper we consider a triple $\left\{U, V, V_{0}\right\}$, where $\{U, V\}$ is an almost unperturbed Schrödinger pair of operators, $\left\{U, V_{0}\right\}$ is a Schrödinger pair of operators, and the pair $\left\{V, V_{0}\right\}$ satisfies the following condition (C) that this pair is almost commuting in the following sense:

$$
\left\|\left[e^{i s V_{0}}, e^{i t V}\right]\right\|_{1} \leq k \min (|s|,|t|), \quad-\infty<s, t<\infty,
$$

where $\|\cdot\|_{1}$ is the trace norm and $k$ is a constant. This triple $\left\{U, V, V_{0}\right\}$ is said to be an almost unperturbed Schrödinger triple satisfying the condition (C). From [5] it is easy to see that if there is a trace class operator $D_{1}$ such that

$$
\left[(V-\lambda I)^{-1},\left(V_{0}-\mu I\right)^{-1}\right]=(V-\lambda I)^{-1}\left(V_{0}-\mu I\right)^{-1} D_{1}\left(V_{0}-\mu I\right)^{-1}(V-\lambda I)^{-1}
$$

for some $\lambda, \mu \in \mathbb{C} \backslash \mathbb{R}$, then (6) holds well.

There are several examples of this kind of triple. Let $\mathscr{H}=L^{2}(\mathbb{R})$,

$$
(Q f)(x)=x f(x) \quad f \in \mathscr{D}(Q),
$$

where $\mathscr{D}(Q)=\left\{f \in L^{2}(\mathbb{R}),(\cdot) f(\cdot) \in L^{2}(\mathbb{R})\right\}$, and

$$
(P f)(x)=i\left(\frac{d}{d x} f\right)(x), \quad f \in \mathscr{D}(P),
$$

where $\mathscr{D}(P)=\left\{f \in L^{2}(\mathbb{R}), f\right.$ is absolutely continuous, and $\left.(\cdot) f(\cdot) \in L^{2}(\mathbb{R})\right\}$. Moreover, let

$$
(V f)(x)=i \frac{d}{d x} f(x)+\frac{\alpha(x)}{2 \pi i} \int \frac{\beta(s) f(s) d s}{x-(s+i o)}+\frac{\overline{\beta(x)}}{2 \pi i} \int \frac{\overline{\alpha(s)} f(s) d s}{x-(s+i o)}
$$

for $f \in \mathscr{D}(P)$, where $\alpha(\cdot)$ and $\beta(\cdot)$ satisfy certain conditions. For example, if $\alpha(x)=c(x-\mu)(x-\lambda)^{-1}$ and $\beta(x)=b \alpha(x)^{-1}$ where $\alpha, \mu \in \mathbb{C} \backslash \mathbb{R}$ and $b, c \in$ $\mathbb{C}$, then $\{Q, P, V\}$ is an almost unperturbed Schrödinger triple of operators satisfying the condition (C). Another example is the triple $\left\{Q, P, \Omega P \Omega^{-1}\right\}$, where $\Omega$ is unitary and $\Omega-I \in \mathscr{L}^{1}$.

In this paper, we study trace formulas for the almost unperturbed Schrödinger triple satisfying the condition (C). A cyclic cocycle is given by the trace formula

$$
\begin{aligned}
\operatorname{tr}( & {\left[e^{i r_{0} U} e^{i s_{0} V_{0}} e^{i t_{0} V}, e^{i r_{1} U} e^{i s_{1} V_{0}} e^{i t_{1} V}\right] } \\
& \left.-\left(e^{-i r_{1}\left(s_{0}+t_{0}\right)}-e^{-i r_{0}\left(s_{1}+t_{1}\right)}\right) e^{i\left(r_{0}+r_{1}\right) U} e^{\left(s_{0}+s_{1}\right) V_{0}} e^{i\left(t_{0}+t_{1}\right) V}\right) \\
= & \operatorname{tr}\left(r_{0}+r_{1}, s_{0}+s_{1}, t_{0}+t_{1}\right)\left(e^{-i r_{1}\left(s_{0}+t_{0}\right) U}-e^{i r_{0}\left(s_{1}+t_{1}\right)}\right)
\end{aligned}
$$

for $\left(r_{0}+r_{1}\right)^{2}+\left(s_{0}+s_{1}+t_{0}+t_{1}\right)^{2} \neq 0$, where $\tau(\cdot, \cdot, \cdot)$ is a continuous function (see Theorem 2). Formula (10) is a refinement of (4). We also prove that there is a function $c(t)$ such that

$$
e^{i t V}=V_{t}+c(t) e^{i t V_{0}}
$$


with

$$
\left(V_{t} f\right)(x)=-\frac{1}{\sqrt{2 \pi}} \int \hat{\tau}(y, y-x, t) f(y) d y,
$$

where $\hat{\tau}(\cdot, s, t)$ is the inverse Fourier transform of $\tau(\cdot, s, t)$ for fixed $s$ and $t$ (see Theorem 3 ). Under certain conditions, this function $c(t) \equiv 1$ (see Theorem 4).

\section{Cyclic Cocycle}

Let $G$ be a Lie group with elements $e^{i k} g(p)$, where $k \in \mathbb{R}$ and $p=$ $(r, s, t) \in \mathbb{R}^{3}$. Suppose the elements in $G$ are subject to the multiplication formula

$$
e^{i k_{1}} g\left(p_{1}\right) e^{i k_{2}} g\left(p_{2}\right)=e^{i\left(k_{1}+k_{2}+\left\langle p_{1}, p_{2}\right\rangle\right)} g\left(p_{1}+p_{2}\right),
$$

where $\left\langle p_{1}, p_{2}\right\rangle=\left(r_{1}\left(s_{2}+t_{2}\right)-r_{2}\left(s_{1}+t_{1}\right)\right) / 2$ for $p_{j}=\left(r_{j}, s_{j}, t_{j}\right), j=1,2$.

For an almost unperturbed Schrödinger triple $\left\{U, V_{0}, V\right\}$ satisfying the condition (C) and the group $G$, define

$$
\rho\left(e^{i k} g(r, s, t)\right)=e^{i(k-r(s+t) / 2)} e^{i r U} e^{i s V_{0}} e^{i t V} .
$$

Then $\left\{\rho\left(e^{i k} g(p)\right): k \in \mathbb{R}, p \in \mathbb{R}^{3}\right\}$ is an "almost" Lie group of unitary operators in the sense of [4], and

$$
\operatorname{tr}\left(\left[\rho\left(h_{1}\right), \rho\left(h_{2}\right)\right]-\left(\rho\left(h_{1} h_{2}\right)-\rho\left(h_{2} h_{1}\right)\right)\right), \quad h_{1}, h_{2} \in G,
$$

is a cyclic one cocycle on $G$. Let

$$
\phi\left(p_{1}, p_{2}\right)=\operatorname{tr}\left(\left[\phi\left(g\left(p_{1}\right)\right), \phi\left(g\left(p_{2}\right)\right)\right]-\phi\left(g\left(p_{1}\right) g\left(p_{2}\right)\right)+\phi\left(g\left(p_{2}\right) g\left(p_{1}\right)\right)\right)
$$

for $p_{j} \in \mathbb{R}^{3}$. Then $\phi(\cdot, \cdot)$ is a locally bounded Baire function satisfying the following conditions:

$$
\begin{gathered}
\phi(0, p)=0, \\
\phi\left(p_{0}, p_{1}\right)=-\phi\left(p_{1}, p_{0}\right),
\end{gathered}
$$

and

$$
\phi\left(p_{1}+p_{2}, p_{0}\right) e^{i\left\langle p_{1}, p_{2}\right\rangle}+\phi\left(p_{2}+p_{0}, p_{1}\right) e^{i\left\langle p_{2}, p_{0}\right\rangle}+\phi\left(p_{0}+p_{1}, p_{2}\right) e^{i\left\langle p_{0}, p_{1}\right\rangle}=0
$$

Now we have to determine the form of $\phi(\cdot, \cdot)$. For fixed $\xi \in \mathbb{R}^{3}$, denote

$$
\eta(p)=\eta(p ; \xi)=\phi(p, \xi-p) \text {. }
$$

Then (12) and (13) imply that

$$
\eta\left(p_{1}+p_{2}\right)=\eta\left(p_{1}\right) e^{i\left\langle p_{2}, \xi\right\rangle}+\eta\left(p_{2}\right) e^{i\left\langle p_{1}, \xi\right\rangle} .
$$

Exchanging $p_{1}$ and $p_{2}$ in (14), we get another equation, and if we eliminate $\eta\left(p_{1}+p_{2}\right)$ from it and (14), we obtain

$$
\eta\left(p_{1}\right)\left(e^{i\left\langle p_{2}, \xi\right\rangle}-e^{-i\left\langle p_{2}, \xi\right\rangle}\right)=\eta\left(p_{2}\right)\left(e^{i\left\langle p_{1}, \xi\right\rangle}-e^{-i\left\langle p_{1}, \xi\right\rangle}\right) .
$$


Let $M(\xi)=\left\{p \in \mathbb{R}^{3}:\langle p, \xi\rangle=0\right\}$. If $M(\xi) \neq \mathbb{R}^{3}$, then choose $p_{2} \in \mathbb{R}^{3} \backslash M(\xi)$ in (15) such that $e^{2 i\left\langle p_{2}, \xi\right\rangle} \neq 1$. Hence, by (15), $\eta(p)=0$ if $e^{2 i\langle p, \xi\rangle}=1$. But if $e^{2 i\langle p, \xi\rangle} \neq 1$, then (15) shows that

$$
\eta(p ; \xi)\left(e^{i\langle p, \xi\rangle}-e^{-\langle p, \xi\rangle}\right)^{-1}
$$

is independent of $p$. Therefore, if $M(\xi) \neq \mathbb{R}^{3}$, there is a number $q(\xi)$ such that

$$
\eta(p ; \xi)=q(\xi)\left(e^{i\langle p, \xi\rangle}-e^{-i\langle p, \xi\rangle}\right), \quad p \in \mathbb{R}^{3} .
$$

The condition $M(r, s, t)=\mathbb{R}^{3}$ is equivalent to $r^{2}+(s+t)^{2}=0$. Thus, if $\left(r_{1}+r_{2}\right)^{2}+\left(s_{1}+s_{2}+t_{1}+t_{2}\right)^{2} \neq 0$, then

$$
\phi\left(p_{1}, p_{2}\right)=q\left(p_{1}+p_{2}\right)\left(e^{i\left\langle p_{1}, p_{2}\right\rangle}-e^{i\left\langle p_{2}, p_{1}\right\rangle}\right) .
$$

Denote $\tau(r, s, t)=q(r, s, t) e^{i r(s+t) / 2}$.

Lemma 1. Let $\left\{U, V_{0}, V\right\}$ be an almost unperturbed Schrödinger triple satisfying the condition $(\mathrm{C})$. Then there is a locally bounded Baire function $\tau(r, s, t)$ defined on $\mathscr{M}=\left\{(r, s, t): r^{2}+(s+t)^{2} \neq 0\right\}$ which is continuous in each variable separately such that

$$
\begin{aligned}
\operatorname{tr} & \left(\left[e^{i r_{0} U} e^{i s_{0} V_{0}} e^{i t_{0} V}, e^{i r_{1} U} e^{i s_{1} V_{0}} e^{i t_{1} V}\right]\right. \\
& \left.-\left(e^{-i r_{1}\left(s_{0}+t_{0}\right)}-e^{-i r_{0}\left(s_{1}+t_{1}\right)}\right) e^{i\left(r_{0}+r_{1}\right) U} e^{i\left(s_{0}+s_{1}\right) V_{0}} e^{i\left(t_{0}+t_{1}\right) V}\right) \\
= & \tau\left(r_{0}+r_{1}, s_{0}+s_{1}, t_{0}+t_{1}\right)\left(e^{-i r_{1}\left(s_{0}+t_{0}\right)}-e^{-i r_{0}\left(s_{1}+t_{1}\right)}\right) .
\end{aligned}
$$

Proof. We have to prove only the separate continuity of $\tau$. Suppose $r \neq 0$. In order to prove that $\tau(r, s, t)$ is a continuous function of $r$, we have to prove only that the left-hand side of $(16)$ is a continuous function of $r_{0}(=r)$ for fixed $r_{1}, s_{0}, t_{0}$ and $t_{1}$, satisfying $r_{1}=0$, and $e^{-i r\left(s_{1}+t_{1}\right)} \neq 1$. It is easy to see that (16) is a sum of the function $\operatorname{tr}\left(e^{i r U} A\right)$ and

$$
q(r)=\operatorname{tr}\left(\left(e^{i r U} e^{i t V}-e^{i r t} e^{i t V} e^{i r U}\right) B\right),
$$

where $t=t_{0}+t_{1}, A \in \mathscr{L}^{1}$, and $B \in \mathscr{L}$ are independent of $r$. It is obvious that $\operatorname{tr}\left(e^{i r U} A\right)$ is a continuous function of $r$. Besides, we have

$$
\begin{aligned}
q(r+\delta)-q(r)= & \operatorname{tr}\left(\left(e^{i \delta U} e^{i t V}-e^{i \delta t} e^{i t V} e^{i \delta U}\right) B e^{i r U}\right) \\
& +\operatorname{tr}\left(\left(e^{i r U} e^{i t V}-e^{i r t} e^{i t V} e^{i r U}\right)\left(e^{i \delta U} e^{i \delta t}-I\right) B\right) .
\end{aligned}
$$

However, from [5, (2)] we have

$$
\left\|e^{i r U} e^{i t V}-e^{i r t} e^{i t V} e^{i r U}\right\|_{1} \leq|r|\|D\|_{1} .
$$

Therefore, $\lim _{\delta \rightarrow 0} q(r+\delta)=q(r)$. Thus $\tau(r, s, t)$ is a continuous function of $r \neq 0$ for fixed $s$ and $t$. Similarly, we may prove the continuity of $\tau$ in the other cases.

In order to analyze the function $\tau$, define functions

$$
\phi(r, s, t)=\operatorname{tr}\left(e^{i r U}\left[e^{i s V_{0}}, e^{i t V}\right]\right)
$$


and

$$
\psi(r, s, t)=\operatorname{tr}\left(\left(e^{i r U} e^{i t V}-e^{i r t} e^{i t V} e^{i r U}\right) e^{i s V_{0}}\right)
$$

on $\mathbb{R}^{3}$. These functions are continuous in each variable separately. In fact, it is obvious that $\phi(r, s, t)$ is a continuous function of $r$ for fixed $s$ and $t$, since $\left[e^{i s V_{0}}, e^{i t V}\right] \in \mathscr{L}^{1}$. To show that $\phi$ is a continuous function of $s$ for fixed $r$ and $t$, we only have to use the formulas

$$
\begin{aligned}
\operatorname{tr}\left(e^{i r U}\left[e^{i(s+\delta) V_{0}}, e^{i t V}\right]\right)= & \operatorname{tr}\left(e^{i r U} e^{i s V_{0}}\left[e^{i \delta V_{0}}, e^{i t V}\right]\right) \\
& +\operatorname{tr}\left(e^{i r U}\left[e^{i s V_{0}}, e^{i t V}\right] e^{i \delta V_{0}}\right)
\end{aligned}
$$

and (6). We omit the details of the proof in ti. ${ }^{-0}$ other cases.

Putting $r_{0}=r, s_{0}=s, t_{0}=r_{1}=s_{1}=0, t_{1}=t$ into (16), we get

$$
\tau(r, s, t)\left(e^{i r t}-1\right)=\phi(r, s, t)+\psi(r, s, t)
$$

for $(r, s, t) \in \mathscr{M}$. If $r=0$ and $s+t \neq 0$, then (17) implies

$$
\operatorname{tr}\left(\left[e^{i s V_{0}}, e^{i t V}\right]\right)=0
$$

for $s+t \neq 0$, since $\psi(0, s, t)=0$. By continuity, (18) holds for $(s, t) \in \mathbb{R}^{2}$. Therefore we get the following theorem as a byproduct of the foregoing analysis:

Theorem 1. Let $\left\{U, V_{0}, V\right\}$ be an almost unperturbed Schrödinger triple satisfying the condition that there is an operator $D_{0} \in \mathscr{L}^{1}$ such that

$$
\left[\left(V-\lambda_{0} I\right)^{-1},\left(V_{0}-\mu_{0} I\right)^{-1}\right]=\left(V_{0}-\mu_{0} I\right)^{-1}\left(V_{0}-\lambda_{0} I\right)^{-1} D_{0}\left(V-\lambda_{0} I\right)^{-1}\left(V_{0}-\mu_{0} I\right)^{-1}
$$

for some $\lambda_{0}, \mu_{0} \in \mathbb{C} \backslash \mathbb{R}$. Then the Pincus principal function for the almost commuting pair of self-adjoint (unbounded) operators $\left\{V_{0}, V\right\}$ equals zero.

Notice that (19) implies (6), so Theorem 1 is a consequence of (18).

Now, we have to obtain an expression for the function $\tau$. Using the Weyl commutation relation (1) of $e^{i r U}$ and $e^{i s V_{0}}$, we may calculate that

$$
\left(1-e^{i r s}\right) \psi(r, s, t)+\left(1-e^{i r(s+t)}\right) \phi(r, s, t)=0 .
$$

Multiplying both sides of $(17)$ by $\left(1-e^{i r s}\right)$ and using (20) to eliminate $\psi$, we obtain

$$
\tau(r, s, t)\left(e^{-i r s}-1\right)=\phi(r, s, t) \quad \text { for }(r, s, t) \in \mathscr{M},
$$

if $e^{\text {irt }} \neq 1$. But both sides of $(21)$ are continuous functions; therefore, $(21)$ is still true even if $e^{i r t}=1$. Thus

(22) $\tau(r, s, t)=\operatorname{tr}\left(e^{i r U}\left[e^{i s V_{0}}, e^{i t V}\right]\right)\left(e^{-i r s}-1\right)^{-1} \quad$ for $(r, s, t) \in \mathscr{M}, e^{i r s} \neq 1$.

If $e^{i r s}=1$ then $\phi(r, s, t)=0$. By the continuity of $\tau$, we have

$$
\tau(r, s, t)=i s^{-1} \frac{\partial}{\partial r} \phi(r, s, t), \quad \text { for }(r, s, t) \in \mathscr{M}, s \neq 0, e^{i r s}=1 .
$$


On the other hand, by $[5,(53)]$ we also have

$$
\tau(r, 0, t)=i r^{-1} \frac{\partial}{\partial s} \phi(r, 0, t) \quad \text { for } r \neq 0,
$$

and

$$
\tau(0,0, t)=\operatorname{tr}\left(D e^{i t V}\right) .
$$

The next step is to examine the case of $M(\xi)=\mathbb{R}^{3}$; i.e., $\xi=(0, s,-s)$. Then (14) becomes

$$
\eta\left(p_{1}+p_{2}\right)=\eta\left(p_{1}\right)+\eta\left(p_{2}\right) .
$$

So, there are functions $K(s)$ and $K_{j}(s), j=1,2$, such that

$$
\eta\left(r_{0}, s_{0}, t_{0}\right)=r_{0} K(s)+s_{0} K_{1}(s)+t_{0} K_{2}(s),
$$

since $\eta(p)$ is a locally bounded Baire function.

Thus $\tau\left(r_{0}, s_{0}, t_{0} ;-r_{0}, s_{1}, t_{1}\right)$ equals

$$
\begin{aligned}
& \operatorname{tr}\left[e^{i r_{0} U} e^{i s_{0} V_{0}} e^{i t_{0} V}, e^{-i r_{0} U} e^{i s_{1} V_{0}} e^{i t_{1} V}\right] \\
& \quad=r_{0} K\left(s_{0}+s_{1}\right)+s_{0} K_{1}\left(s_{0}+s_{1}\right)+t_{0} K_{2}\left(s_{0}+s_{1}\right),
\end{aligned}
$$

provided that $s_{0}+s_{1}+t_{0}+t_{1}=0$. If $r_{0}=0$, then, from Theorem 1, we have $s_{0} K_{1}\left(s_{0}+s_{1}\right)+t_{0} K_{2}\left(s_{0}+s_{1}\right)=0$. Hence

$$
r K(s)=\operatorname{tr}\left(\left[e^{i r U} e^{i s V_{0}} e^{-i s V}, e^{-i r U}\right]\right) .
$$

Therefore, by $[5,(58)]$,

$$
K(s)=i \operatorname{tr}\left(\left[\left(U, e^{-i s V}\right]+s e^{-i s V}\right) e^{-i s V_{0}}\right) .
$$

Theorem 2. Let $\left\{U, V_{0}, V\right\}$ be an almost unperturbed Schrödinger triple satisfying the condition (C). If $\left(r_{0}+r_{1}\right)^{2}+\left(s_{0}+s_{1}+:_{0}+t_{1}\right)^{2} \neq 0$, then (16) holds, where the function $\tau(r, s, t)$ is defined by (22)-(25). If $\left(r_{0}+r_{1}\right)^{2}+\left(s_{0}+s_{1}+t_{0}+t_{1}\right)^{2}=0$, then the left-hand side of (16) equals $r_{0} K\left(s_{0}+s_{1}\right)$, where the function $K(\cdot)$ is defined by (27).

By continuity, (20) and (21) imply that

$$
\psi(r, s, t)=\left(e^{i r t}-e^{-i r s}\right) \tau(r, s, t), \quad \text { for } r^{2}+(s+t)^{2} \neq 0 .
$$

Lemma 2. Let $\left\{U, V_{0}, V\right\}$ be an almost unperturbed Schrödinger triple of operators satisfying the condition (C). If $\left(r_{1}+r_{2}\right)^{2}+\left(s_{1}+s_{2}+t\right)^{2} \neq 0$, then

$$
\begin{gathered}
\operatorname{tr}\left(e^{i r_{1} U} e^{i s_{1} V_{0}}\left(e^{i r_{2} U} e^{i s_{2} V_{0}} e^{i t V}-e^{i r_{2} t} e^{i t V} e^{i r_{2} U} e^{i s_{2} V_{0}}\right)\right) \\
\quad=\left(e^{i\left(r_{2} t-r_{1} s_{2}\right)}-e^{-i s_{1} r_{2}}\right) \tau\left(r_{1}+r_{2}, s_{1}+s_{2}, t\right) .
\end{gathered}
$$

Proof. Denote the left-hand side of (29) by $\psi\left(r_{1}, r_{2}, s_{1}, s_{2}, t\right)$. It is easy to see that

$$
\psi\left(r_{1}, r_{2}, s_{1}, s_{2}, t\right)=e^{-i s_{1} r_{2}} \phi\left(r_{1}+r_{2}, s_{1}, s_{2}, t\right)+e^{-i r_{1} s_{2}} \psi\left(r_{1}, r_{2}, s_{1}+s_{2}, t\right),
$$


where

$$
\dot{\varphi}\left(r, s_{1}, s_{2}, t\right)=\operatorname{tr}\left(e^{i r U} e^{i s_{1} V_{0}}\left[e^{i s_{2} V_{0}}, e^{i t V}\right]\right)
$$

and

$$
\psi\left(r_{1}, r_{2}, s, t\right)=\operatorname{tr}\left(e^{i r_{1} U} e^{i s V_{0}}\left(e^{i r_{2} U} e^{i t V}-e^{i r_{2} t} e^{i t V} e^{i r_{2} U}\right)\right)
$$

We may calculate that

$$
\psi\left(r_{1}, r_{2}, s, t\right)+e^{-i\left(r_{2} s+r_{1} t\right)} \psi\left(r_{2}, r_{1}, s, t\right)=e^{-i r_{1} t} \psi\left(r_{1}+r_{2}, s, t\right) .
$$

Eliminating $\psi$ from (31) and using another equation obtained from (31) by exchanging $r_{1}$ and $r_{2}$, we get

$$
\psi\left(r_{1}, r_{2}, s, t\right)=\left(e^{i r_{2} t}-e^{-i r_{2} s}\right) \tau\left(r_{1}+r_{2}, s, t\right)
$$

if $e^{i\left(r_{1}+r_{2}\right)(s+t)} \neq 1$. However, both sides of (32) are continuous functions. Therefore (32) holds even if $e^{i\left(r_{1}+r_{2}\right)(s+t)}=1$. Similarly, we have

$$
\phi\left(r, s_{1}, s_{2}, t\right)=\left(e^{-i r s_{2}}-1\right) \tau\left(r, s_{1}+s_{2}, t\right) .
$$

Equation (29) now follows from (30), (32), and (33).

\section{A LEMMA}

For $\xi \in L^{2}(\mathbb{R})$, let $\widehat{\xi}$ be the inverse Fourier transform of $\xi$.

Lemma 3. Let $\{Q, P\}$ be the Schrödinger pair of operators on $L^{2}(\mathbb{R})$ defined by (8) and (9), let $A \in \mathscr{L}^{1}$, and let $\xi, \eta \in L^{2}(\mathbb{R}) \cap L^{1}(\mathbb{R})$. Then

$$
\frac{1}{2 \pi} \int\left(\iint \operatorname{tr}\left(e^{i(r-p) Q} e^{i s P} A\right) \widehat{\xi}(r) \overline{\hat{\eta}(p)} e^{i p s} d r d p\right) d s=(A \xi, \eta) .
$$

Proof. For $f \in L^{2}(\mathbb{R})$, the vector-valued integral

$$
L(s ; f)=\frac{1}{2 \pi} \iint e^{i(r-p) Q} e^{i s P} f \widehat{\xi}(r) \overline{\hat{\eta}(p)} e^{i p s} d r d p
$$

converges in $L^{2}(\mathbb{R})$. As a function of $x$, the vector $L(s ; f)$ is

$$
\frac{1}{2 \pi} \iint e^{i x r} f(x-s) \widehat{\xi}(r) \hat{\eta}(p) e^{i(x-s) p} d r d p=f(x-s) \xi(x) \overline{\eta(x-s)},
$$

for a.e.x .

Therefore, for every $h \in L^{2}(\mathbb{R})$, we have

$$
(L(s ; f), h)=\int f(x-s) \xi(x) \overline{\eta(x-s) h(x)} d x .
$$

Hence, the weak integral $\int L(s ; f) d s$ exists and

$$
\left(\int L(s ; f) d s, h\right)=(f, \eta)(\xi, h) \text {. }
$$

Thus

$$
\int L(s ; f) d s=(f, \eta) \xi
$$


Without loss of generality, we may assume $A \geq 0$. Let $\left\{e_{k}\right\}$ be an orthonormal basis satisfying $A e_{k}=\lambda_{k} e_{k}, \lambda_{k} \geq 0$ and $\sum \lambda_{k}<+\infty$. It is obvious that

$$
\frac{1}{2 \pi} \iint \operatorname{tr}\left(e^{i(r-p) Q} e^{i s P} A\right) \widehat{\xi}(r) \overline{\hat{\eta}(p)} e^{i r s} d r d p=\sum \lambda_{k}\left(L\left(s ; e_{k}\right), e_{k}\right) .
$$

From (35) and (36), (34) follows.

For fixed $s_{2}$ and $t, \phi\left(r, s_{1}, s_{2}, t\right)$ is a continuous function of $\left(r, s_{1}\right)$. Therefore, by (33), it is easy to see that $\tau(r, s, t)$ is a continuous function of $(r, s)$ for fixed $t$.

\section{ForM OF $e^{i t V}$}

In this section, an expression for $e^{i t V}$ is given by using $\tau(x, s, t)$. Let $K$ be the space of all indefinitely differentiable functions with compact supports endowed with the topology defined in [2] and [3] and let $K^{\prime}$ be the space of all linear continuous functionals (distributions) on $K$. Let $Z$ be the space of Fourier transforms of the functions in $K$, and $Z^{\prime}$ be the space of the Fourier transforms of the distributions in $K^{\prime}$. It is obvious that the function $\tau(\cdot, s, t) \in K$ for fixed $s$ and $t$ and the function $\psi\left(\cdot, r_{2}, s_{1}, s_{2}, t\right) \in K$ for fixed $r_{2}, s_{1}, s_{2}$, and $t$. Let $\hat{\tau}(\cdot, s, t) \in Z^{\prime}$ and $\hat{\psi}\left(\cdot, r_{2}, s_{2}, t\right) \in Z^{\prime}$ be the inverse Fourier transforms of $\tau(\cdot, s, t)$ and $\psi\left(\cdot, r_{2}, s_{1}, s_{2}, t\right)$, respectively. Then, by (29),

$$
\hat{\psi}\left(x, r_{2}, s_{1}, s_{2}, t\right)=\hat{\tau}\left(x+s_{2}, s_{1}+s_{2}, t\right) e^{i r_{2}\left(x+s_{2}+t\right)}-\hat{\tau}\left(x, s_{1}+s_{2}, t\right) e^{i r_{2}\left(x-s_{1}\right)} .
$$

Let

$$
A(r, s, t)=e^{i r t} e^{i t V} e^{i r U} e^{i s V_{0}}-e^{i r U} e^{i s V_{0}} e^{i t V} .
$$

Without loss of generality, we may assume that $\mathscr{H}=L^{2}(\mathbb{R}, \mathscr{D}), U=Q$, and $V_{0}=P$. For $\xi, \eta \in K$, we obtain by (34)

$$
\begin{aligned}
(A(r, s, t) \xi, \eta)= & \frac{1}{(2 \pi)^{3 / 2}} \int\left(\int \int \int e ^ { i ( r _ { 1 } - p ) x } \left(\hat{\tau}\left(x, s_{1}+s, t\right) e^{i r\left(x-s_{1}\right)}\right.\right. \\
& \left.\left.-\hat{\tau}\left(x+s, s_{1}+s, t\right) e^{i r(x+s+t)}\right) \hat{\xi}\left(r_{1}\right) \overline{\hat{\eta}(p)} e^{i p s_{1}} d r_{1} d p d x\right) d s_{1} .
\end{aligned}
$$

Therefore

$$
\begin{aligned}
(A(r, s, t) \xi)(x)= & \frac{1}{\sqrt{2 \pi}} \int \hat{\tau}(y, y-x+s, t) e^{i r x} \xi(y) d y \\
& -\frac{1}{\sqrt{2 \pi}} \int \hat{\tau}(y+s, y-x+s, t) e^{i r(y+s+t)} \xi(y) d y .
\end{aligned}
$$

Define an operator $V_{t}$ :

$$
\left(V_{t} \xi\right)(x)=-\frac{1}{\sqrt{2 \pi}} \int \hat{\tau}(y, y-x, t) \xi(y) d y, \quad \xi \in K .
$$

Then (37) shows that

$$
\left(e^{i r U} e^{i s V_{0}} e^{-i t V_{0}}\left(V_{t}-e^{i t V}\right)-e^{-i t V_{0}}\left(V_{t}-e^{i t V}\right) e^{i r U} e^{i s V_{0}}\right) \xi=0
$$


for all $r, s, t \in \mathbb{R}$ and $\xi \in K$. Therefore the operator $e^{-i t V_{0}}\left(V_{t}-e^{i t V}\right)$ commutes $e^{i r U} e^{i s V_{0}}$ for $r, s \in \mathbb{R}$. Thus, there is a function $c(t)$ such that

$$
e^{i t V}=V_{t}+c(t) e^{i t V_{0}} \text {. }
$$

Theorem 3. Let $\{Q, P, V\}$ be an almost unperturbed Schrödinger triple of operators on $L^{2}(\mathbb{R})$ satisfying $(C)$, and let $V_{t}$ be the operator defined by (38). Then there is a function $c(t)$ such that (39) holds. If $V$ is not of the form $V=P+\alpha I$, $\alpha \in \mathbb{C}$, then the function $c(t)$, satisfying the condition that $\left\{V_{t}+c(t) e^{i t P}: t \in \mathbb{R}\right\}$ is a one-parameter group of operators, is unique.

Thus the operator $V$ is determined by the function $\tau(r, s, t)$ if the almost unperturbed Schrödinger triple $\left\{U, V_{0}, V\right\}$ satisfies the condition that $U$ is simple.

Proof. We have to prove only the uniqueness of $c(t)$. From the equation

$$
\left(V_{t_{1}}+c\left(t_{1}\right) e^{i t_{1} P}\right)\left(V_{t_{2}}+c\left(t_{2}\right) e^{i t_{2} P}\right)=V_{t_{1}+t_{2}}+c\left(t_{1}+t_{2}\right) e^{i\left(t_{1}+t_{2}\right) P},
$$

it is easy to see that

(40)

$$
\begin{aligned}
\frac{1}{2 \pi} \int \hat{\tau}\left(v-u+t_{2}, z-u-t_{1}, t_{1}\right) \hat{\tau}\left(v, u-t_{2}, t_{2}\right) d u \\
\quad-\frac{1}{\sqrt{2 \pi}} c\left(t_{1}\right) \hat{\tau}\left(v, z-t_{2}, t_{2}\right)-\frac{1}{\sqrt{2 \pi}} c\left(t_{2}\right) \hat{\tau}\left(v+t_{2}, z-t_{1}, t_{1}\right)+c\left(t_{1}\right) c\left(t_{2}\right) \delta(z) \\
=-\frac{1}{\sqrt{2 \pi}} \hat{\tau}\left(v, z-t_{1}-t_{2}, t_{1}+t_{2}\right)+c\left(t_{1}+t_{2}\right) \delta(z) .
\end{aligned}
$$

Suppose $b(\cdot)$ is another function satisfying the condition that $\left\{V_{t}+b(t) e^{i t P}\right.$ : $t \in \mathbb{R}\}$ is also a one-parameter group. Then (40) implies that

$$
\begin{aligned}
& \frac{1}{\sqrt{2 \pi}}\left(b\left(t_{1}\right)-c\left(t_{1}\right)\right) \hat{\tau}\left(v, z-t_{2}, t_{2}\right)+\frac{1}{\sqrt{2 \pi}}\left(b\left(t_{2}\right)-c\left(t_{2}\right)\right) \hat{\tau}\left(v+t_{2}, z-t_{1}, t_{1}\right) \\
& \quad=\left(c\left(t_{1}+t_{2}\right)-b\left(t_{1}+t_{2}\right)-c\left(t_{1}\right) c\left(t_{2}\right)+b\left(t_{1}\right) b\left(t_{2}\right)\right) \delta(z) .
\end{aligned}
$$

Exchanging the roles of $t_{1}$ and $t_{2}$ in (41), we obtain the result that, if $b\left(t_{j}\right)-$ $c\left(t_{j}\right) \neq 0 \quad j=1,2$, then

$$
\tau_{1}\left(v, t_{2}\right)+\tau_{1}\left(v+t_{2}, t_{1}\right)=\tau_{1}\left(v, t_{1}\right)+\tau_{1}\left(v+t_{1}, t_{2}\right),
$$

where

$$
\tau_{1}(v, t)=\hat{\tau}(v, z-t, t)(b(t)-c(t))^{-1}
$$

for $t$ satisfying $b(t) \neq c(t)$ and fixed $z$. Taking the Fourier transform of both sides of (42), we may conclude that there is a function $\tau(x, z)$ such that

$$
\tau(x, s, t)=\left(1-e^{-i x t}\right)(b(t)-c(t)) \tau(x, s+t) .
$$

It is easy to see that if $b(\cdot) \neq c(\cdot)$ then there exists a sequence $\left\{t_{n}\right\}$ satisfying $b\left(t_{n}\right) \neq c\left(t_{n}\right)$. From (41) and (43), there is a number $c_{n}$ such that 
$\hat{\tau}\left(y, z-2 t_{n}, 2 t_{n}\right)=c_{n} \delta(z)$. Thus $V_{2 t_{n}}=-\frac{1}{\sqrt{2 \pi}} c_{n} e^{i 2 t_{n} P}$. Hence $V-P$ is a multiple of identity. This proves the theorem.

Theorem 4. Let $\{Q, P, V\}$ be an almost unperturbed Schrödinger triple of operators on $L^{2}(\mathbb{R})$ satisfying the condition $(\mathrm{C})$. If $e^{i t V}-e^{i t P} \in \mathscr{L}^{1}$ for $t \in \mathbb{R}$, then the function $c(t)$ in (39) is the constant 1.

Proof. Let

$$
\tau_{0}(r, s, t)=\operatorname{tr}\left(e^{i r Q} e^{i s P}\left(e^{i t P}-e^{i t V}\right)\right) .
$$

Then it is easy to calculate that

$$
\psi\left(r_{1}, r_{2}, s_{1}, s_{2}, t\right)=\left(e^{i\left(r_{2} t-r_{1} s_{2}\right)}-e^{-i s_{1} r_{2}}\right) \tau_{0}\left(r_{1}+r_{2}, s_{1}+s_{2}, t\right) .
$$

Comparing equations (29) and (41), we have

$$
\tau=\tau_{0}
$$

Since $e^{i t V}-e^{i t V_{0}} \in \mathscr{L}^{1}$ is a product of two Hilbert-Schmidt operators, it is easy to see that there is a kernel $k(x, y, t)$ such that

$$
\left(\left(e^{i t V}-e^{i t P}\right) f\right)(x)=\frac{1}{\sqrt{2 \pi}} \int k(y, x, t) f(y) d y
$$

and

$$
\operatorname{tr}\left(e^{i r Q} e^{i s P}\left(e^{i t V}-e^{i t P}\right)\right)=\frac{1}{\sqrt{2 \pi}} \int e^{i r x} k(x, x-s, t) d x .
$$

Therefore, $\hat{\tau}_{0}(x, s, t)=-k(x, x-s, t)$ or $k(y, x, t)=-\hat{\tau}_{0}(y, y-x, t)$. From (46) and (47) it follows that $e^{i t V}=V_{t}+e^{i t P}$, and the theorem is proved.

\section{ACKNOWLEDGMENT}

The author wishes to express his appreciation to Professor Paul S. Muhly for his valuable suggestions in organizing this paper and [6].

\section{REFERENCES}

1. R. W. Carey and J. D. Pincus, Almost commuting pairs of unitary operators and flat currents, Integral Equations Operator Theory 4 (1981), 45-122.

2. I. M. Gelfand and G. E. Shilov, Generalized functions, Academic Press, New York, 1964.

3. L. Schwartz, Introduction to the theory of distributions, Canad. Math. Cong. Ser. No. 1, 1952.

4. D. Xia, Trace formula for almost Lie group of operators and cyclic cocycles, Integral Equations Operator Theory 9 (1986), 570-587.

5. __ On the almost unperturbed Schrödinger pair of operators, Integral Equations Operator Theory 12 (1989), 242-279.

6. __ Principal distribution for almost unperturbed Schrödinger pair of operators, Proc. Amer. Math. Soc. (to appear). 